\title{
Factores individuales y contextuales relacionados con el binge drinking en adolescentes españoles: un enfoque multinivel
} Individual and contextual factors related to binge drinking

\author{
Ester Teixidó-Compañó*,**,***; Luis Sordo**********; Marina Bosque-Prous* ,**,***; Susanna \\ Puigcorbé*; Gregorio Barrio******; M. Teresa Brugal****; María José J. Belza**** ,******; \\ Albert EsPeLt *,**,****,*******.
}

* Agència de Salut Pública de Barcelona. Barcelona, España; **Instituto de Investigación Biomédica Sant Pau (IIB Sant Pau). Barcelona, España; ***Universidad Pompeu Fabra (UPF). Barcelona, España; ****CIBER de Epidemiología y Salud Pública (CIBERESP). Madrid, España; *****Departamento de Medicina Preventiva y Salud Pública. Facultad de Medicina. Universidad Complutense de Madrid. Madrid, España; ******Escuela Nacional de Sanidad. Instituto de Salud Carlos III. Madrid, España; ********Facultat de Ciències de la Salut de Manresa, Universitat de Vic Universitat Central de Catalunya (UVicUCC), Av. Universitària, 46, 08242 Manresa, España.

\section{Resumen}

El objetivo de este estudio era estimar la prevalencia de binge drinking por provincias en España y estimar el efecto de variables individuales y contextuales relacionadas con dicho consumo en adolescentes españoles. Se realizó un estudio transversal con datos de la Encuesta sobre uso de drogas en Enseñanzas Secundarias en España (ESTUDES 2014) a estudiantes de 14 a 18 años $(\mathrm{N}=34.259)$. La variable dependiente fue binge drinking en adolescentes durante los últimos 30 días. Las variables independientes individuales fueron variables socioeconómicas y variables relacionadas con el acceso y la disponibilidad de alcohol. Las variables contextuales fueron el consumo de alcohol en adultos, políticas públicas relacionadas con el alcohol y factores socioeconómicos. Se ajustaron modelos de regresión de Poisson multinivel con variancia robusta, obteniendo razones de prevalencia (RP) y sus intervalos de confianza al $95 \%$.

Los resultados muestran que la prevalencia de binge drinking en estudiantes españoles en función de la provincia era similar para ambos sexos $(r=0,72)$. A nivel individual, el binge drinking se asociaba principalmente a una percepción de acceso fácil al alcohol (RP: 1,38; IC 95\%: 1,23-1,55), a su consumo en zonas abiertas [(RP: 3,82; IC 95\%: 3,44-4,24) < una vez al mes y (RP: 6,57; IC 95\%: 5,85-7,37) $\geq$ una vez al mes], a tener uno de los dos padres que permite beber (RP: 1,42; IC 95\%: 1,37-1,47), y a disponer de más de 30 euros semanales (RP: 1,51; IC 95\%: 1,37-1,67). Las variables contextuales no se asociaban al binge drinking cuando se consideraban las variables individuales. En conclusión, el binge drinking se asociaba con variables individuales relacionadas con una alta accesibilidad y disponibilidad de alcohol independientemente de las variables contextuales. Estas variables explicaban la variabilidad de el binge drinking entre las provincias.

Palabras clave: Alcohol; Consumo intensivo; Adolescentes; Accesibilidad al alcohol.

\section{Abstract}

The aim of this study was to estimate the prevalence of binge drinking by regions in Spain and assess the effect of individual and contextual factors related to this drinking pattern in adolescents. A cross-sectional study was performed with data from the 2014 Spanish School Survey on Drug Use (ESTUDES) in students aged 14-18 years $(\mathrm{N}=34,259)$. The outcome was binge drinking in adolescents during the last 30 days. Individual independent variables were socioeconomic variables and variables related to access to alcohol and its availability. Contextual variables consisted of adult alcohol consumption, public policies on alcohol, and socioeconomic factors. Multilevel Poisson regression models with robust variance were estimated, obtaining prevalence ratios (PR) and their $95 \%$ confidence intervals.

The results showed that the prevalence of youth binge drinking by region of residence was similar for both sexes $(r=0.72)$. At the individual level, binge drinking was mainly associated with the perception of easy access to alcohol (PR: 1.38; 95\% CI: 1.23-1.55), consumption in open areas [(PR: 3.82; 95\% CI: 3.44-4.24) < once a month and (PR: 6.57; 95\% CI: 5.85-7.37) $\geq$ once a month], at least one parent allowing alcohol consumption (PR: 1.42; 95\% CI: 1.371.47 ), and receiving $>30$ euros weekly (PR :1.51; 95\% CI: $1.37-1.67$ ). Contextual variables were not associated with youth binge drinking when individual variables were considered. In conclusion, youth binge drinking was associated with individual variables related to high alcohol accessibility and availability, regardless of contextual variables. These variables explained the variability in binge drinking among Spanish regions.

Keywords: Alcohol; Underage drinking; Binge drinking; Adolescents; Alcohol accessibility. 
$\mathrm{E}$ 1 consumo de alcohol es uno de los factores principales de riesgo de mortalidad y morbilidad a nivel mundial (Rehm et al., 2009; Shield et al., 2013; OMS, 2014). Aunque puede afectar a cualquier grupo de edad, la población adolescente es particularmente vulnerable (CNAPA, 2014; Zeigler et al., 2005). La adolescencia es un periodo durante el cual se inician muchas conductas de riesgo, incluyendo la ingesta de alcohol (Pitkänen, Lyyra, y Pulkkinen, 2005; Plan Nacional Sobre Drogas, 2016). El patrón de consumo de alcohol de riesgo más frecuente entre adolescentes es el binge drinking. El binge drinking se define como el consumo de al menos 60 gramos o más de alcohol puro en al menos una ocasión en los últimos 30 días (CNAPA, 2014) o el consumo de 5 o más bebidas alcohólicas en una sola ocasión de consumo, e.g., en un intervalo aproximado de dos horas (Plan Nacional Sobre Drogas, 2016). La prevalencia de binge drinking mensual entre las y los adolescentes europeos varía entre 13\%-56\% (en España 32,2\%) (Hibell et al., 2012; Plan Nacional Sobre Drogas, 2016). A esta edad, el binge drinking se asocia con otras conductas de riesgo, como conducir bajo los efectos del alcohol, comportamientos violentos o prácticas sexuales de riesgo (CNAPA, 2014; Font-Ribera et al., 2013; Kuntsche et al., 2013). También hay evidencia que el consumo de alcohol en la adolescencia puede ser un factor de riesgo de consumo elevado y problemático de alcohol en la etapa adulta (Pitkänen, et al, 2005; Pitkänen, Kokko, Lyyra, y Pulkkinen, 2008).

Varios estudios indican que el binge drinking en la adolescencia está asociado con variables individuales, como la edad, el sexo, el nivel socioeconómico de la familia, la estructura familiar, los hábitos y actitudes de miembros de la familia y amigos hacia el alcohol, la permisividad, la supervisión y el control de los padres, y la cantidad de dinero para gastos personales (Heimisdottir, Vilhjalmsson, Kristjansdottir, y Meyrowitsch, 2010; Llorens, Barrio, Sánchez, Suelves, y ESTUDES Grupo de Trabajo, 2011). La influencia de las personas adultas también puede ser otro factor, dado que éstas son modelos para la juventud (Bendtsen et al., 2014; Heimisdottir et al., 2010). Las personas adultas pueden tolerar o fomentar condiciones familiares o del entorno que facilitan el consumo de alcohol entre las y los jóvenes (Foley, Altman, Durant, y Wolfson, 2004; Heimisdottir et al., 2010; Reimuller, Shadur, y Hussong, 2011; van der Vorst, Engels, Meeus, Deković, y Van Leeuwe, 2005) o incluso directamente ofrecerles o darles alcohol (Foley et al., 2004; Jones-Webb et al., 1997; Pettigrew, Pescud, Jarvis, y Webb, 2013). En España, el fenómeno del botellón, que consiste en grupos que consumen alcohol en espacios públicos abiertos, tales como plazas o parques, se ha convertido en una práctica generalizada en muchas regiones, y también se ha asociado con el binge drinking (Cortés, Espejo, Martín del Río, y Gómez, 2010; Romo-Avilés, Marcos-Marcos, Marquina-Márquez, y Gil-García, 2016).
No obstante, el consumo de alcohol y los problemas relacionados con éste también pueden explicarse por otras variables contextuales: (1) políticas públicas relacionadas con el alcohol, como las restricciones de acceso al alcohol, impuestos sobre el alcohol, políticas de seguridad vial, o la regulación de publicidad sobre el alcohol (Bendtsen et al., 2014; Nelson et al., 2013, 2005; Paschall, Grube, y Kypri, 2009; OMS, 2014; Xuan et al., 2013, 2015); (2) factores socioeconómicos y demográficos, como cambios en los ingresos per cápita o la tasa de desempleo (Krieg y Kuhl, 2016; Pedersen, Bakken, y von Soest, 2015); y (3) consumo de alcohol por parte de adultos a nivel poblacional (Bendtsen et al., 2014; Nelson, Naimi, Brewer, y Nelson, 2009; Nelson, Naimi, Brewer, y Wechsler, 2005; Xuan et al., 2013).

Aunque tanto variables individuales como contextuales se han asociado al consumo de alcohol, la mayoría de estudios sobre el consumo de alcohol entre la población joven se han centrado en las características individuales de las y los adolescentes (Nelson et al., 2005) o han explorado la relación entre el consumo de jóvenes y el de adultos a nivel familiar (Rossow, Keating, Felix, y McCambridge, 2015). De este modo, faltan estudios sobre el consumo de alcohol entre adolescentes que incluyan variables individuales y contextuales relacionadas tanto con factores socioeconómicos y demográficos, como con la accesibilidad y la disponibilidad de alcohol. Dados estos antecedentes, es importante valorar el efecto de algunas variables contextuales sobre el binge drinking en un país como España, donde este fenómeno ha aumentado de manera significativa en los últimos años (Galán, González, y Valencia-Martín, 2014), y que ha sufrido una crisis económica profunda desde el año 2008, unida a unas tasas de desempleo elevadas.

El objetivo de este estudio fue estimar la prevalencia de binge drinking por provincias en España, y valorar el efecto de los factores individuales y contextuales relacionados con este patrón de consumo de alcohol en adolescentes.

\section{Métodos}

\section{Fuentes de datos}

Estudio transversal con datos individuales extraídos de la encuesta española ESTUDES 2014 (acrónimo de la Encuesta sobre uso de drogas en Enseñanzas Secundarias en España), implementada en el marco del Plan Nacional sobre Drogas. La muestra estaba compuesta de 34.259 estudiantes de 14 a 18 años de edad ( 17.498 chicas; 16.761 chicos) que asisten en centros educativos de secundaria de todas las regiones de España, incluyendo escuelas urbanas y rurales, públicas y privadas. La muestra de estudiantes representaba, aproximadamente, el $70 \%$ de la población joven de este rango de edad en España. El muestreo fue por conglomerados bietápico, seleccionando aleatoriamente los centros educativos como unidades de primera etapa, y las aulas como unidades de segunda etapa. La descripción detalla- 
da de la muestra se ha publicado con anterioridad (Plan Nacional sobre Drogas, 2016). El alumnado para el que no había información sobre su país de nacimiento $(0,1 \%)$, el consumo de alcohol en espacios públicos abiertos $(4,7 \%)$, el control parental $(3 \%)$ o haber hecho binge drinking $(1,2 \%)$ fue excluido. Por lo contrario, las y los estudiantes para los cuales no había información sobre el nivel de estudios alcanzado por los padres $(14,7 \%)$, el dinero para gastos personales recibido semanalmente $(8,2 \%)$, la permisividad de los padres hacia el consumo de alcohol (16,9\%), y el acceso percibido al alcohol $(13,8 \%)$ fueron incluidos en la categoría "desconocido" debido a su elevado porcentaje. Para las demás variables independientes no se encontraron valores faltantes. Los datos agregados o contextuales medidos a nivel de provincia en España se tomaron de varias fuentes, como las encuestas españolas EDADES (acrónimo español para la Encuesta sobre alcohol y drogas en España) de los años 2011 y 2013, que compila información sobre el consumo de alcohol per cápita en adultos y sobre las políticas que limitan el consumo de alcohol en espacios públicos abiertos; la encuesta ESTUDES, que recopila datos sobre las políticas de prevención de venta de alcohol a menores; y la encuesta EPA del año 2011 (acrónimo español para la Encuesta de Población Activa), que ofrece datos respecto a la tasa de desempleo.

\section{Variables}

\section{Variable dependiente}

La variable de respuesta fue la ocurrencia de binge drinking entre las y los estudiantes de 14-18 años, definido como el consumo de cinco o más unidades de bebida estándar en un intervalo de 2 horas, al menos una vez en los últimos 30 días, para ambos sexos. Solamente se consideraba la ocurrencia de binge drinking al menos una vez en los últimos 30 días, sin la posibilidad de saber si era un patrón continuo en el tiempo.

\section{Variables individuales socioeconómicas}

Las variables socioeconómicas incluían el nivel socioeconómico, la edad (como variable continua), el sexo, el país de nacimiento (definiendo a la persona nacida fuera de España como "inmigrante"), y la provincia de residencia en España (52 regiones; siendo Palencia la provincia con el menor número de estudiantes $[\mathrm{n}=50]$ y Madrid con el mayor número $[\mathrm{n}=4.634]$ ). El nivel socioeconómico se midió según el nivel de estudios alcanzado (nivel más alto de estudios completados por el padre o la madre) (Krieger, Williams, y Moss, 1997).

\section{Variables individuales relacionadas con la accesibili- dad y la disponibilidad de alcohol}

Las variables independientes relacionadas con el acceso y la disponibilidad individual al alcohol fueron la percep- ción de acceso al alcohol, el consumo de alcohol en espacios públicos abiertos, el dinero para gastos personales disponible semanalmente, la permisividad de los padres hacia el consumo de alcohol, y el control parental. La percepción de las y los jóvenes de acceso al alcohol se obtuvo a través de la pregunta "¿Qué dificultad piensas que tendrías para conseguir bebidas alcohólicas?”, con las cuatro posibles respuestas agrupadas en dos categorías (difícil, fácil). Se obtuvo información sobre el consumo de alcohol en espacios públicos abiertos, conocido en España como botellón, a través de la pregunta "En los últimos 12 meses, ¿con qué frecuencia has hecho un botellón?”, con las siete posibles respuestas agrupadas en tres categorías ( $\geq$ una vez/mes, < una vez/mes, nunca). El dinero para gastos personales recibido semanalmente se obtuvo a través de una pregunta abierta, clasificada en 4 categorías ( 0 euros, $<10$ euros, entre 10-30 euros y $>30$ euros). La permisividad de los padres hacia el consumo de alcohol se obtuvo a través de la pregunta "Si quisieras tomar bebidas alcohólicas, ¿tus padres te permitirían hacerlo?”, considerando permisividad parental cuando al menos uno de ambos permitiría el consumo de alcohol dentro o fuera del hogar. El control parental hace referencia a la frecuencia en la que los padres saben con quién y a dónde salen sus hijos/as por las noches, con cinco posibles respuestas agrupadas en tres categorías (a menudo, a veces, raramente).

\section{Variables contextuales socioeconómicas y relaciona- das con el alcohol}

Las variables independientes contextuales medidas a nivel de provincia en España fueron el consumo de alcohol per cápita en la población adulta, las políticas públicas relacionadas con el alcohol, y los factores socioeconómicos. El consumo de alcohol per cápita en la población adulta se estimó como la media de gramos de alcohol puro consumido diariamente en la población de 25-64 años residente en cada provincia, a partir de las preguntas sobre cantidad-frecuencia de la encuesta EDADES, que hacen referencia a los últimos 30 días. Los gramos de alcohol puro se obtuvieron multiplicando el volumen de bebidas alcohólicas ingeridas por la graduación de cada categoría de bebida y por 0,79 (densidad de alcohol en $\mathrm{g} / \mathrm{ml}$ ). Respecto a las políticas públicas relacionadas con el alcohol, la prevalencia de estudiantes que percibían un acceso fácil al alcohol, se cuantificó como un indicador de la efectividad de las políticas regionales sobre reglamentos e intervenciones para prevenir la venta de alcohol a menores, agrupando los resultados individuales de la encuesta ESTUDES en cada provincia. La prevalencia de consumo de alcohol en los espacios abiertos de uso público, el botellón, se cuantificó como un indicador de la efectividad de las políticas sobre reglamentos e intervenciones para limitar el consumo de alcohol en espacios públicos abiertos. Se consideró la prevalencia de botellón en los últimos 12 meses en la pobla- 
ción española de 15-30 años a partir de la encuesta EDADES. El factor socioeconómico seleccionado fue la tasa de desempleo entre la población activa de 16-64 años, como la media de los cuatro trimestres de 2011 en cada provincia, según la encuesta EPA en España del 2011.

\section{Análisis estadístico}

Los resultados de la encuesta se ponderaron por Comunidad Autónoma, titularidad de la escuela, y tipo de estudios para corregir la desproporcionalidad de la muestra respecto al marco muestral. Primeramente, se hizo una descripción de la muestra, separando por sexo, para estimar la prevalencia de binge drinking según las covariables individualizadas, con sus intervalos de confianza del $95 \%$ (IC 95\%). Para determinar la distribución del binge drinking en las diferentes provincias españolas, se crearon dos mapas en función del sexo, que permitieron clasificar las diferentes provincias según el cuartil al cual pertenecían. Para explorar la asociación entre el binge drinking en las y los jóvenes y los factores contextuales, se realizaron correlaciones lineales simples por provincia, con sus respectivos gráficos de dispersión. Por último, para identificar el efecto de los factores individuales y contextuales sobre el binge drinking entre adolescentes, se construyeron modelos de regresión multinivel de Poisson con varianza robusta, obteniendo Razones de Prevalencia (RP) con sus IC del 95\% (Espelt, Mari-Dell'Olmo, Penelo, y Bosque-Prous, 2017). El modelo inicial (modelo 0) incluía únicamente el binge drinking entre jóvenes para calcular su variabilidad entre las diferentes provincias españolas. A continuación, se estimó la asociación entre las variables individuales o contextuales con el binge drinking, creando un modelo multinivel con las variables socioeconómicas individuales (modelo 1), otro con las variables de acceso y disponibilidad individual al alcohol (modelo 2), otro con todas las variables individuales juntas (modelo 3), y otro modelo que contenía todas las variables contextuales (modelo 4). Finalmente, se ajustó un modelo multivariado (modelo 5) para estimar el efecto conjunto de los factores individuales y contextuales sobre el binge drinking en jóvenes, asumiendo que la intersección tenía un efecto aleatorio. Todos los modelos se construyeron conjuntamente para chicas y chicos debido al tamaño muestral limitado en algunas provincias y a la similitud de la distribución del binge drinking según las variables independientes para ambos sexos. El análisis se llevó a cabo mediante el paquete estadístico STATA, versión 14.

\section{Resultados}

El $89 \%$ de las chicas y el $90 \%$ de los chicos de la muestra de estudio habían nacido en España, aproximadamente la mitad de ellos/as tenían padres con estudios secundarios o menos (53\% de las chicas; $49 \%$ de los chicos) y más del $90 \%$ tenían menos de 18 años. La prevalencia total de binge drinking entre jóvenes en el último mes fue del 33\%. Para ambos sexos, la prevalencia de binge drinking aumentaba con la edad, y aunque en general la prevalencia era más alta para los chicos, las diferencias entre los dos sexos eran significativas a partir de los 17 años. Para ambos sexos, la prevalencia de binge drinking era más elevada entre españoles que entre inmigrantes ( $32 \%$ vs. $28 \%$ en chicas, y $34 \%$ vs. $32 \%$ en chicos), entre aquellos cuyos padres tenían estudios secundarios o menos, comparado con los que tenían estudios universitarios (34\% vs. $30 \%$ en chicas, y $37 \%$ vs. $33 \%$ en chicos), y entre aquellos con al menos uno de los padres que permitía el consumo de alcohol, comparado con los que tenían padres no lo permitían (49\% vs. $19 \%$ en chicas, y $53 \%$ vs. $19 \%$ en chicos). Respecto al control parental, cuando los padres sabían con quién y a dónde salían sus hijos/as por las noches, la prevalencia de binge drinking era menor en ambos sexos. Asimismo, declararon haber realizado binge drinking en los últimos 30 días más del $40 \%$ de las y los adolescentes que disponían de más de 30 euros/semana, más del 35\% de los que percibían un acceso fácil al alcohol, y más del $65 \%$ de los que habían bebido alcohol en espacios públicos abiertos en el último mes (Tabla 1).

La figura 1 muestra la distribución del binge drinking, separadamente por chicas y chicos, según provincia de residencia. Se obtuvo un coeficiente de correlación entre sexos de 0,72. La prevalencia de binge drinking fue superior en las provincias del centro de España, exceptuando Madrid, que estaba en el cuartil más bajo para ambos sexos.

Todas las variables individuales mostraron una asociación significativa con el binge drinking, independientemente de si los efectos se midieron por separado o conjuntamente con las variables contextuales. Respecto a las variables socioeconómicas individuales, ser chica (RP 0,95; IC 95\% 0,91-0,99) y tener padres con estudios universitarios vs. estudios secundarios o menos (RP 0,95; IC 95\% 0,91-0,99) tuvo un efecto protector, mientras que tener una mayor edad estaba asociado a un mayor riesgo (RP 1,35; IC 95\% 1,31-1,40). Por otro lado, cuando se analizaron las variables socioeconómicas por separado, haber nacido en España se asoció a un menor riesgo de binge drinking (RP 0,88; IC $95 \%$ 0,82-0,95), pero cuando se incluyeron otras variables en el modelo, fuesen individuales o contextuales, se observó una asociación en la dirección opuesta (RP 1,13; IC $95 \%$ 1,08-1,18). Respecto a las variables individuales relacionadas con el alcohol, se halló un aumento del riesgo de binge drinking para el acceso fácil al alcohol (RP 1,40; IC 95\% 1,25-1,58), consumo de alcohol en espacios públicos abiertos (RP 6,77; IC 95\% 6,01-7,63 al menos una vez al mes), permisividad de los padres (RP 1,52; IC 95\% 1,451,59), control parental (RP 1,17; IC 95\% 1,12-1,22 cuando los padres rara vez sabían con quién salía su hijo/a por las noches y RP 1,14; IC 95\% 1,07-1,20 cuando los padres rara vez sabían a dónde salía su hijo/a por las noches), y dinero 
Ester Teixidó-Compañó, Luis Sordo, Marina Bosque-Prous, Susanna Puigcorbé, Gregorio Barrio,

M. Teresa Brugal, María José Belza, Albert Espelt

Tabla 1. Prevalencia de binge drinking en los últimos 30 días e intervalo de confianza del $95 \%$ (IC 95\%) por sexo y otras variables individuales entre estudiantes de 14-18 años. España, 2014

\begin{tabular}{|c|c|c|c|c|c|c|c|c|}
\hline \multirow[b]{2}{*}{$\mathrm{N}$ total $=34.259$} & \multirow{2}{*}{$\begin{array}{c}\text { Chicas } \\
n\end{array}$} & \multicolumn{3}{|c|}{$\begin{array}{c}\text { Binge } \\
\text { drinking }\end{array}$} & \multicolumn{2}{|l|}{ Chicos } & \multicolumn{2}{|c|}{$\begin{array}{c}\text { Binge } \\
\text { drinking }\end{array}$} \\
\hline & & $\%$ & $\%$ & IC 95\% & n & $\%$ & $\%$ & IC 95\% \\
\hline Total & 17.498 & 51,1 & 31,6 & $(30,9-32,3)$ & 16.761 & 48,9 & 34,0 & $(33,3-34,7)$ \\
\hline \multicolumn{9}{|l|}{ Edad } \\
\hline 14 años & 3.517 & 20,1 & 14,8 & $(13,7-16,0)$ & 3.143 & 18,8 & 13,6 & $(12,5-14,9)$ \\
\hline 15 años & 4.423 & 25,3 & 25,4 & $(24,1-26,7)$ & 4.130 & 24,6 & 24,3 & $(23,0-25,7)$ \\
\hline 16 años & 4.221 & 24,1 & 37,3 & $(35,9-38,8)$ & 3.928 & 23,4 & 38,2 & $(36,7-39,8)$ \\
\hline 17 años & 4.080 & 23,3 & 41,9 & $(40,4-43,4)$ & 4.039 & 24,1 & 47,6 & $(46,0-49,1)$ \\
\hline 18 años & 1.258 & 7,2 & 47,9 & $(45,2-50,7)$ & 1.521 & 9,1 & 55,3 & $(52,8-57,8)$ \\
\hline \multicolumn{9}{|l|}{ País de nacimiento } \\
\hline España & 15.533 & 88,8 & 32,0 & $(31,3-32,8)$ & 15.062 & 89,9 & 34,2 & $(33,5-35,0)$ \\
\hline Fuera de España & 1.965 & 11,2 & 28,1 & $(26,1-30,1)$ & 1.700 & 10,1 & 32,1 & $(29,9-34,3)$ \\
\hline \multicolumn{9}{|l|}{ Nivel de estudios de los padres } \\
\hline Estudios secundarios o menos & 9.327 & 53,3 & 34,0 & $(33,0-34,9)$ & 8.143 & 48,6 & 36,6 & $(35,6-37,7)$ \\
\hline Universitarios & 5.874 & 33,6 & 29,5 & $(28,3-30,6)$ & 5.991 & 35,7 & 32,9 & $(31,7-34,1)$ \\
\hline Desconocido & 2.297 & 13,1 & 27,4 & $(25,6-29,3)$ & 2.627 & 15,7 & 28,4 & $(26,7-30,2)$ \\
\hline \multicolumn{9}{|l|}{ Acceso percibido al alcohol } \\
\hline Difícil & 1.269 & 7,3 & 10,0 & $(8,4-11,8)$ & 1.233 & 7,4 & 13,2 & $(11,5-15,3)$ \\
\hline Fácil & 14.453 & 82,6 & 35,5 & $(34,8-36,3)$ & 13.377 & 79,8 & 38,7 & $(37,9-39,5)$ \\
\hline Desconocido & 1.775 & 10,1 & 14,9 & $(13,3-16,6)$ & 2.151 & 12,8 & 16,5 & $(15,0-18,2)$ \\
\hline \multicolumn{9}{|l|}{ Consumo de alcohol en espacios públicos y abiertos } \\
\hline No & 7.604 & 43,5 & 7,7 & $(7,1-8,3)$ & 7.858 & 46,9 & 8,1 & $(7,6-8,8)$ \\
\hline く una vez/mes & 5.312 & 30,4 & 34,9 & $(33,7-36,2)$ & 4.808 & 28,7 & 40,9 & $(39,5-42,3)$ \\
\hline$\geq$ una vez/mes & 4.582 & 26,2 & 67,3 & $(66,0-68,7)$ & 4.096 & 24,4 & 75,5 & $(74,1-76,8)$ \\
\hline \multicolumn{9}{|l|}{ Permisividad de los padres } \\
\hline Ninguno de los padres permite el consumo de alcohol & 7.815 & 44,7 & 19,1 & $(18,2-19,9)$ & 7.010 & 41,8 & 18,6 & $(17,7-19,5)$ \\
\hline Al menos uno de los padres permite el consumo de alcohol & 7.067 & 40,4 & 48,8 & $(47,6-50,0)$ & 6.916 & 41,3 & 52,9 & $(51,7-54,0)$ \\
\hline Desconocido & 2.616 & 15,0 & 22,6 & $(21,1-24,3)$ & 2.835 & 16,9 & 26,1 & $(24,6-27,8)$ \\
\hline \multicolumn{9}{|l|}{ Los padres saben con quién sale el/la adolescente por las noches } \\
\hline A menudo & 14.769 & 84,4 & 28,9 & $(28,1-29,6)$ & 12.742 & 76,0 & 31,5 & $(30,7-32,3)$ \\
\hline A veces & 1.633 & 9,3 & 44,6 & $(42,2-47,0)$ & 2.104 & 12,6 & 41,2 & $(39,1-43,3)$ \\
\hline Raramente & 1.096 & 6,3 & 48,9 & $(46,0-51,9)$ & 1.915 & 11,4 & 42,8 & $(40,6-45,1)$ \\
\hline \multicolumn{9}{|l|}{ Los padres saben a dónde sale el/la adolescente por las noches } \\
\hline A menudo & 14.156 & 80,9 & 28,5 & $(27,8-29,3)$ & 12.015 & 71,7 & 30,53 & $(29,7-31,4)$ \\
\hline A veces & 1.930 & 11,0 & 42,6 & $(40,4-44,8)$ & 2.412 & 14,4 & 41,52 & $(39,6-43,5)$ \\
\hline Raramente & 1.412 & 8,1 & 47,5 & $(44,9-50,1)$ & 2.334 & 13,9 & 44,03 & $(42,0-46,0)$ \\
\hline \multicolumn{9}{|l|}{ Dinero disponible semanal } \\
\hline 0 euros & 1.869 & 10,7 & 17,9 & $(16,2-19,7)$ & 1.948 & 11,6 & 16,9 & $(15,3-18,6)$ \\
\hline Menos de 10 euros & 3.742 & 21,4 & 25,0 & $(23,6-26,4)$ & 3.098 & 18,5 & 24,4 & $(23,0-26,0)$ \\
\hline Entre $10-30$ euros & 9.401 & 53,7 & 36,9 & $(36,0-37,9)$ & 8.871 & 52,9 & 40,0 & $(39,0-41,0)$ \\
\hline Más de 30 euros & 1.093 & 6,3 & 43,8 & $(40,9-46,8)$ & 1.525 & 9,1 & 48,1 & $(45,6-50,6)$ \\
\hline Desconocido & 1.393 & 8,0 & 22,4 & $(20,3-24,7)$ & 1.319 & 7,9 & 25,1 & $(22,8-27,5)$ \\
\hline
\end{tabular}

Nota. *Excluyendo a los estudiantes para los cuales no había datos respecto a su país de nacimiento, consumo de alcohol en espacios públicos abiertos o participación en binge drinking. ESTUDES: Encuesta sobre uso de drogas en enseñanzas secundarias en España. 
(a) Prevalencia de binge drinking en chicas

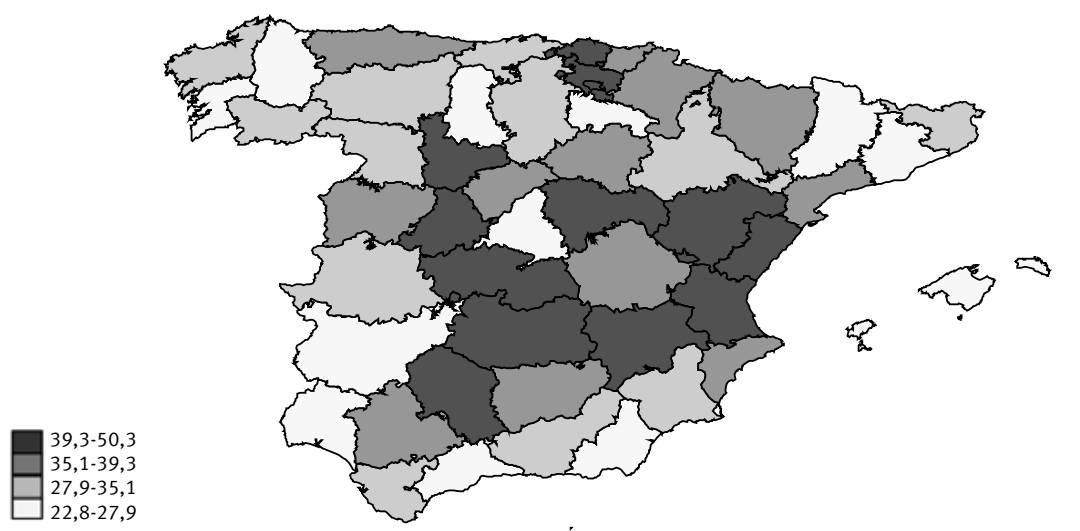

(b) Prevalencia de binge drinking en chicos

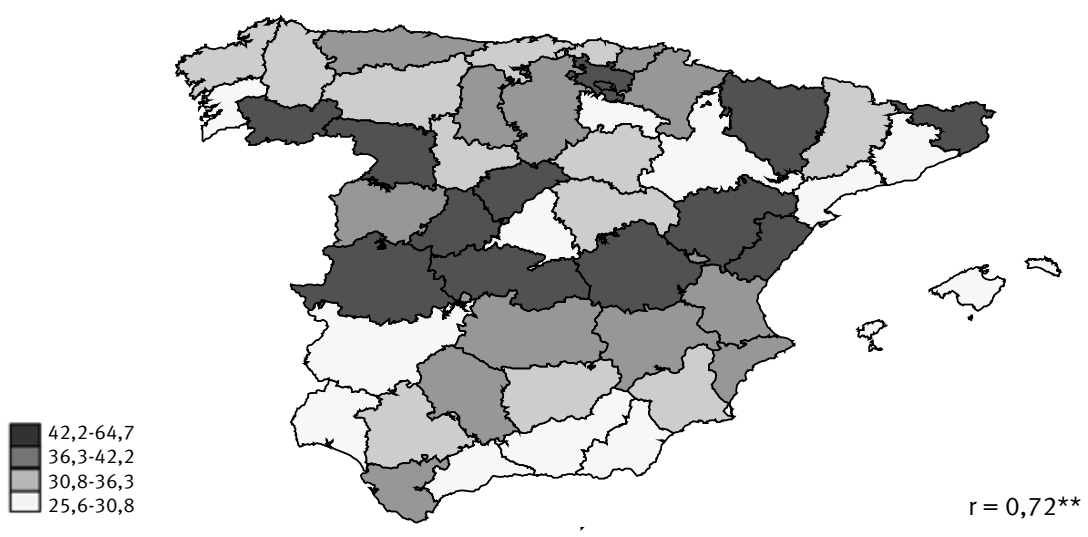

Nota. *El mapa excluyó Las Palmas, Santa Cruz de Tenerife, Ceuta y Melilla. La prevalencia de binge drinking en estas provincias fue: $21,4 \%$ (chicas) y $20,8 \%$ (chicos) en Las Palmas; $28,7 \%$ (chicas) y 30,4\% (chicos) en Santa Cruz de Tenerife; $5,5 \%$ (chicas) y 11,9\% (chicos) en Ceuta; y 10,8\% (chicas) y $16,4 \%$ (chicos) en Melilla. ** Coeficiente de correlación entre la prevalencia de binge drinking en chicos y chicas en las diferentes provincias.

$\star * \star$ Varianza de la prevalencia de binge drinking entre provincias: chicas $=3,3 \%$; chicos $=3,1 \%$.

Figura 1. Prevalencia de binge drinking en los últimos 30 días por provincias de España y sexo, en estudiantes de 14-18 años. España, 2014

disponible cada semana (RP 1,56; IC 95\% 1,42-1,73 de más de 30 euros). Asimismo, se observó un gradiente en la asociación en las últimas tres variables (Tabla 2).

Respecto a las variables contextuales, como muestra la Figura 2, el consumo de alcohol per cápita en adultos y la prevalencia de adolescentes que perciben un acceso fácil al alcohol mostraron las correlaciones positivas más fuertes con el binge drinking entre jóvenes de ambos sexos (r(chicas $)=0,304$; valor $\mathrm{p}=0,03 ; \mathrm{r}($ chicos $)=0,230$; valor $\mathrm{p}=$ 0,$10 ; \mathrm{y} \mathrm{r}$ (chicas) $=0,603$; valor $\mathrm{p}<0,001 ; \mathrm{r}($ chicos $)=0,585$; valor $\mathrm{p}<0,001)$, respectivamente), mientras que la tasa de desempleo mostró una correlación negativa, especialmente en los chicos $(r$ (chicos $)=-0,305$; valor $p=0,03)$. No obstante, en los modelos multinivel (Tabla 2), solo el acceso fácil al alcohol tuvo una asociación significativa con el binge drinking y solo cuando se consideraron las variables contextuales de forma aislada de las individuales (RP 1,03; IC 95\% 1,02-1,04). De hecho, el modelo vacío determinó que la variabilidad en el binge drinking entre las y los jóvenes de diferentes provincias fue aproximadamente del 3,6\%.
Cuando se consideraron todas las variables individuales (modelo 3), éstas explicaron el $75 \%$ de la variabilidad en el binge drinking. A pesar de esto, el modelo 2, que contenía únicamente las variables individuales relacionadas con el alcohol, explicó el 78\%. Cuando se consideraron las variables contextuales únicamente, el modelo explicó el $46 \%$ de la variabilidad en el binge drinking (modelo 4). Por último, al considerar tanto las variables individuales como las contextuales, el poder explicativo del modelo fue del $77 \%$.

\section{Discusión}

\section{Principales resultados}

La prevalencia de binge drinking en los últimos 30 días entre estudiantes españoles de 14-18 años fue del 32\% en chicas y del $34 \%$ en chicos, con variaciones según provincia de residencia. Aunque la prevalencia era más alta en chicos que en chicas, hubo una elevada correlación entre sexos en la prevalencia de bringe drinking según la provincia de residencia en España. A nivel individual, las principales 
Ester Teixidó-Compañó, Luis Sordo, Marina Bosque-Prous, Susanna Puigcorbé, Gregorio Barrio,

M. Teresa Brugal, María José Belza, Albert Espelt

Tabla 2. Efecto de factores individuales y contextuales sobre el binge drinking en los últimos 30 días entre adolescentes de 14-18 años. España, 2014

\begin{tabular}{|c|c|c|c|c|c|c|c|c|c|c|}
\hline \multirow{2}{*}{$\begin{array}{l}\text { BINGE DRINKING } \\
\text { Factores individuales }\end{array}$} & \multirow{2}{*}{\multicolumn{2}{|c|}{$\begin{array}{c}\text { Modelo } 1 \\
\text { (variables socioeconómicas) }\end{array}$}} & \multicolumn{2}{|c|}{$\begin{array}{c}\text { Modelo } 2 \\
\text { (variables relacionadas } \\
\text { con el alcohol) }\end{array}$} & \multicolumn{2}{|c|}{$\begin{array}{c}\text { Modelo } 3 \\
\text { (todas las variables } \\
\text { individuales) }\end{array}$} & \multicolumn{2}{|c|}{$\begin{array}{c}\text { Modelo } 4 \\
\text { (variables } \\
\text { contextuales) } \\
\end{array}$} & \multicolumn{2}{|c|}{$\begin{array}{c}\text { Modelo } 5 \\
\text { (multifactorial) }\end{array}$} \\
\hline & & & $\mathrm{RP}$ & IC 95\% & $\mathrm{RP}$ & IC 95\% & $\mathrm{RP}$ & IC 95\% & $\mathrm{RP}$ & IC 95\% \\
\hline Edad & 1,35 & $(1,31-1,40)$ & & & 1,09 & $(1,06-1,12)$ & & & 1,09 & $(1,06-1,12)$ \\
\hline \multicolumn{11}{|l|}{ Sexo } \\
\hline Chicos & 1 & & & & 1 & & & & 1 & \\
\hline Chicas & 0,95 & $(0,91-0,99)$ & & & 0,93 & $(0,90-0,96)$ & & & 0,93 & $(0,90-0,97)$ \\
\hline \multicolumn{11}{|l|}{ País de nacimiento } \\
\hline España & 1 & & & & 1 & & & & 1 & \\
\hline Fuera de España & 0,88 & $(0,82-0,95)$ & & & 1,13 & $(1,08-1,19)$ & & & 1,13 & $(1,08-1,18)$ \\
\hline \multicolumn{11}{|l|}{ Nivel de estudios de los padres } \\
\hline Estudios secundarios o menos & 1 & & & & 1 & & & & 1 & \\
\hline Universitarios & 0,95 & $(0,91-0,99)$ & & & 0,96 & $(0,92-0,99)$ & & & 0,95 & $(0,92-0,99)$ \\
\hline \multicolumn{11}{|c|}{ Acceso percibido al alcohol por parte del/de la adolescente } \\
\hline Difícil & & & 1 & & 1 & & & & 1 & \\
\hline Fácil & & & 1,40 & $(1,25-1,58)$ & 1,38 & $(1,23-1,54)$ & & & 1,38 & $(1,23-1,55)$ \\
\hline \multicolumn{11}{|c|}{ Consumo de alcohol en espacios abiertos } \\
\hline No & & & 1 & & 1 & & & & 1 & \\
\hline « una vez/mes & & & 3,90 & $(3,50-4,34)$ & 3,82 & $(3,44-4,24)$ & & & 3,82 & $(3,44-4,24)$ \\
\hline$\geq$ una vez/mes & & & 6,77 & $(6,01-7,63)$ & 6,56 & $(5,85-7,36)$ & & & 6,57 & $(5,85-7,37)$ \\
\hline \multicolumn{11}{|l|}{ Permisividad de los padres } \\
\hline Ninguno de los padres permite & onsumo o & cohol & 1 & & 1 & & & & 1 & \\
\hline Al menos uno de los padres pe & e el const & de alcohol & 1,52 & $(1,45-1,59)$ & 1,42 & $(1,37-1,47)$ & & & 1,42 & $(1,37-1,47)$ \\
\hline A veces & & & 1,10 & $(1,04-1,16)$ & 1,09 & $(1,04-1,15)$ & & & 1,10 & $(1,04-1,16)$ \\
\hline Raramente & & & 1,17 & $(1,12-1,22)$ & 1,16 & $(1,10-1,22)$ & & & 1,16 & $(1,10-1,22)$ \\
\hline \multicolumn{11}{|c|}{ Los padres saben a dónde sale el/la adolescente por las noches } \\
\hline A menudo & & & 1 & & 1 & & & & 1 & \\
\hline A veces & & & 1,07 & $(1,03-1,12)$ & 1,07 & $(1,03-1,12)$ & & & 1,07 & $(1,03-1,12)$ \\
\hline Raramente & & & 1,14 & $(1,07-1,20)$ & 1,15 & $(1,09-1,22)$ & & & 1,15 & $(1,09-1,22)$ \\
\hline \multicolumn{11}{|l|}{ Dinero disponible semanal } \\
\hline 0 euros & & & 1 & & 1 & & & & 1 & \\
\hline Menos de 10 euros & & & 1,10 & $(1,00-1,22)$ & 1,13 & $(1,02-1,25)$ & & & 1,13 & $(1,02-1,25)$ \\
\hline Entre $10-30$ euros & & & 1,30 & $(1,19-1,43)$ & 1,29 & $(1,18-1,42)$ & & & 1,29 & $(1,18-1,41)$ \\
\hline Más de 30 euros & & & 1,56 & $(1,42-1,73)$ & 1,52 & $(1,38-1,67)$ & & & 1,51 & $(1,37-1,67)$ \\
\hline \multicolumn{11}{|l|}{ Factores socioeconómicos } \\
\hline Tasa de desempleo & & & & & & & 1,01 & $(0,97-1,05)$ & 0,98 & $(0,95-1,01)$ \\
\hline \multicolumn{11}{|c|}{ Proxies de la efectividad de políticas públicas relacionadas con el control del alcohol } \\
\hline Prevalencia de adolescentes qu & erciben $\mathrm{u}$ & il acceso al alco & hol & & & & 1,03 & $(1,02-1,04)$ & 1,00 & $(0,99-1,01)$ \\
\hline Prevalencia de adolescentes qu & onsumen & hol en espacios & abiertos & & & & 1,00 & $(1,00-1,01)$ & 1,00 & $(1,00-1,00)$ \\
\hline \multicolumn{11}{|c|}{ Consumo de alcohol per cápita en adultos } \\
\hline Gramos de alcohol puro/día & & & & & & & 1,00 & $(0,99-1,02)$ & 1,00 & $(0,99-1,01)$ \\
\hline Varianza & & 0,0370 & & 0,0080 & & 0,0083 & & 0,0196 & & 0,0081 \\
\hline
\end{tabular}



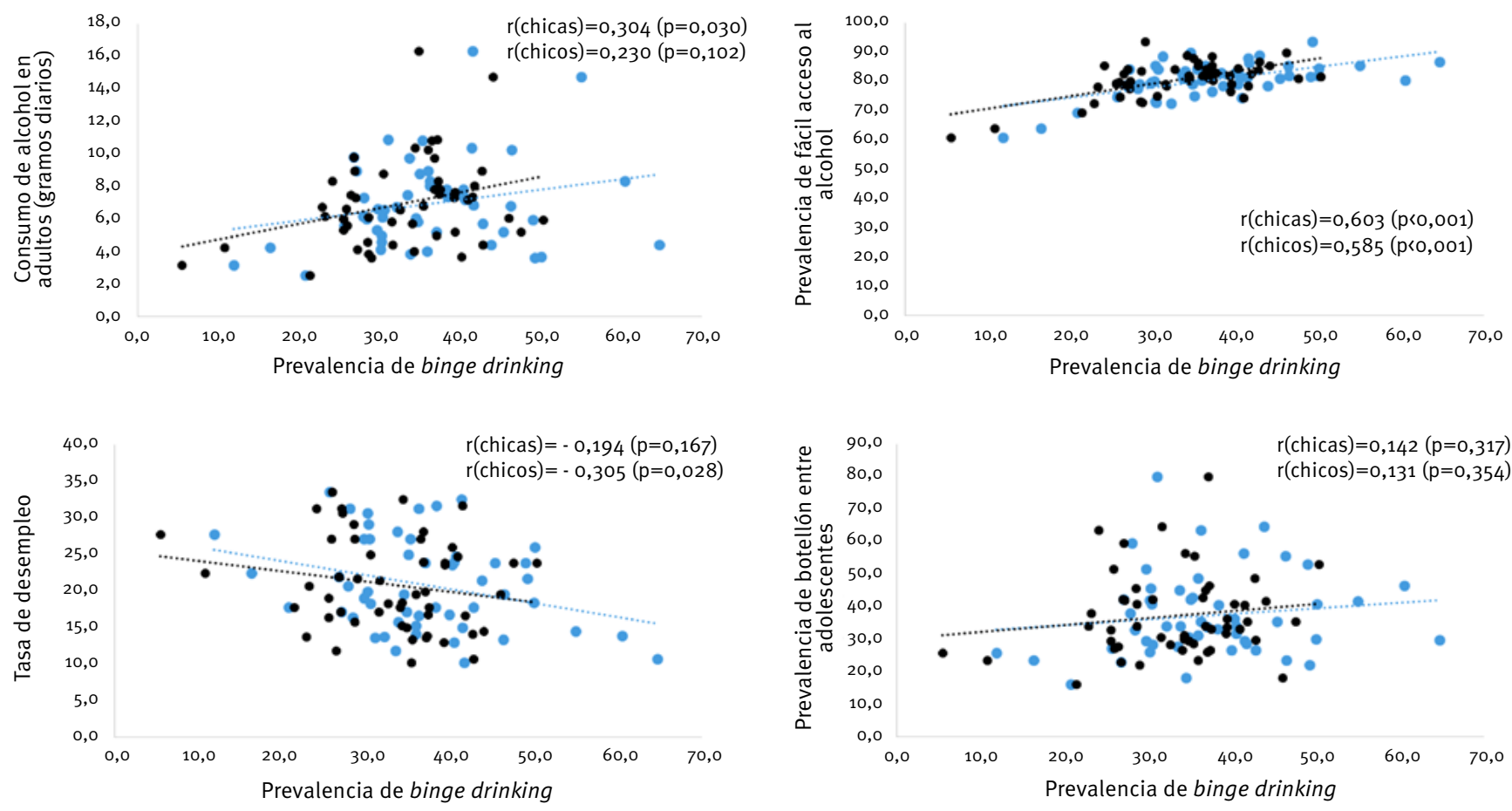

- Chicas • Chicos

Figura 2. Correlación entre el binge drinking en adolescentes y el consumo de alcohol per cápita en adultos, tasa de desempleo e indicadores de políticas públicas regionales sobre alcohol

variables asociadas con un mayor riesgo de binge drinking entre adolescentes eran la percepción de acceso fácil al alcohol, participar en botellones -especialmente en el último mes-, mayor permisividad de los padres, menor control parental, y el dinero semanal disponible para gastos personales. Las provincias con una proporción más elevada de adolescentes que percibían un acceso fácil al alcohol tenían una mayor prevalencia de binge drinking entre las y los jóvenes. No obstante, esta asociación desaparecía cuando se tenían en cuenta las variables individuales.

En nuestro estudio, la prevalencia general de binge drinking en los últimos 30 días entre estudiantes españoles de 14-18 años fue del 33\%. Este resultado es similar a los obtenidos por otros estudios en Europa y en Estados Unidos, con prevalencias de binge drinking en jóvenes de entre 26\%-47\% (CNAPA, 2014; Hibell et al., 2012; Llorens et al., 2011; Nelson et al., 2009; Nelson et al., 2005; Xuan et al., 2013). No obstante, la prevalencia de binge drinking parece haber disminuido, en comparación con la observada en la encuesta ESTUDES 2012, que indicaba una prevalencia de binge drinking del 42\% (Plan Nacional sobre Drogas, 2016). Según el Plan Nacional sobre Drogas, esta disminución puede explicarse por la reducción del consumo de alcohol entre estudiantes de 14-15 años, y coincide con un aumento en la percepción de los riesgos del consumo de alcohol, en comparación con la encuesta del año 2012.
Las variables individuales relacionadas con el alcohol eran las que mejor predecían las diferencias observadas en el binge drinking en adolescentes entre las provincias españolas. Por lo tanto, independientemente de las variables contextuales, se encontró una mayor prevalencia de binge drinking entre adolescentes que percibían un acceso fácil al alcohol, habían hecho botellón en el último mes, tenían al menos uno de los padres que permitía el consumo de alcohol, cuyos padres raramente sabían a dónde ni con quién salían por las noches, y que disponían de dinero semanal para gastos personales. Estudios anteriores ya han sugerido que la disponibilidad de dinero semanal y el acceso fácil al alcohol entre las y los adolescentes aumenta la probabilidad de consumo de alcohol y de binge drinking (Jones y Magee, 2014; Llorens et al., 2011). También existe evidencia que la permisividad y el control de los padres podrían estar asociados con el consumo de alcohol entre adolescentes (Abar, Abar, y Turrisi, 2009; Foley et al., 2004; Heimisdottir et al., 2010; Llorens et al., 2011; Nash, McQueen, y Bray, 2005; van der Vorst et al., 2005). Concretamente, parece que imponer reglas estrictas y desaprobar por completo el consumo de alcohol de las y los adolescentes previene el consumo abusivo de alcohol y las consecuencias negativas relacionadas con éste (Abar et al., 2009; van der Vorst et al., 2005). En este sentido, un estudio encontró que aquellos/as adolescentes que declaraban que sus padres desaprobaban con contundencia el consumo de alcohol, 
también reportaban tener un ambiente familiar positivo, con mayor control por parte de los padres o mejor comunicación, que podía atenuar el efecto potencialmente negativo de los compañeros y compañeras sobre su consumo de alcohol, y podía aumentar su autoeficacia para rechazar el alcohol (Nash et al., 2005). Nuestro estudio, de forma similar a otros estudios en España, encontró una asociación entre el botellón y el binge drinking entre jóvenes (Cortés et al., 2010; Romo-Avilés et al., 2016). Las razones que pueden explicar el consumo elevado de alcohol durante el botellón incluyen centrarse en los efectos positivos del consumo de alcohol (como divertirse o facilitar las relaciones sociales), la presión entre iguales para beber alcohol, el mayor acceso al alcohol, y las expectativas de las y los adolescentes que sus amigos/as les vigilarán y cuidarán si beben grandes cantidades de alcohol (Espejo, Cortés, del Río, Giménez, y Gómez, 2012; Gómez, Fernández, Romero, y Luengo, 2008; Romo-Avilés et al., 2016).

De todas maneras, las variables contextuales explicaron casi la mitad de la variabilidad observada entre provincias españolas. De manera específica, cuanto mayor era la proporción de jóvenes que percibían un acceso fácil al alcohol a nivel provincial, mayor era el riesgo de binge drinking en esa provincia. Este resultado es coherente con la evidencia que muestra una asociación entre políticas de control del alcohol más restrictivas y una menor ingesta de alcohol por parte de la población adolescente (Nelson et al., 2005; Xuan et al., 2015). No obstante, esta asociación desaparecía cuando se tenían en cuenta las variables individuales. Varias razones pueden explicar este resultado. Primero, tal y como reportó el informe ESPAD (Hibell et al., 2012), la mayoría de estudiantes europeos perciben un acceso fácil al alcohol ( $81 \%$ de estudiantes en el informe ESPAD y en este estudio), aunque muchos países tienen leyes que restringen el acceso de la población adolescente al alcohol. Por lo tanto, es posible que, a pesar de la similitud entre las leyes en las diferentes provincias españolas, haya una gran variedad en el cumplimiento de las mismas. Segundo, podría ser que la variable contextual utilizada en este estudio tenga una asociación fuerte con el acceso y la disponibilidad individual al alcohol, porque es una variable individual agregada. Por último, otra posibilidad es que debido a que la variabilidad de las variables contextuales medidas en nuestro estudio entre provincias era muy pequeña, el factor más influente en el binge drinking entre adolescentes fuese el acceso individual de cada adolescente al alcohol.

Sorprendentemente, en este estudio no hubo una asociación entre el consumo de alcohol per cápita en adultos y el binge drinking entre adolescentes. No obstante, otros estudios han mostrado que el consumo de alcohol entre adultos a nivel poblacional, sí que tiene un efecto sobre el consumo de alcohol entre adolescentes (Bendtsen et al., 2014; Nelson et al., 2009; Nelson et al., 2005; Xuan et al., 2013, 2015). Una posible explicación de la falta de sig- nificación en la asociación del consumo de alcohol entre adultos y adolescentes, podría ser la variable utilizada para medir el consumo de alcohol en adultos. Esta variable fue extraída de una encuesta poblacional y quizás no refleja adecuadamente la distribución del consumo de alcohol en las diferentes provincias.

\section{Fortalezas y limitaciones}

Hasta donde sabemos, este es el primer estudio en España que analiza la influencia de diversas variables individuales y contextuales sobre el binge drinking en adolescentes a nivel de provincia. Una de las principales fortalezas de este estudio es el uso de una metodología multinivel para analizar el fenómeno del binge drinking en adolescentes, lo que permite tener en cuenta simultáneamente el efecto de factores individuales y contextuales. Además, también se usó una encuesta poblacional representativa de estudiantes españoles de 14-18 años. No obstante, esta encuesta excluye a la población adolescente que está fuera del sistema educativo formal. Por este motivo, se realizó un análisis de sensibilidad que incluyó únicamente a estudiantes de 14-16 años; los resultados no mostraron diferencias significativas, en comparación con los obtenidos de la muestra íntegra del estudio.

La principal limitación de este estudio es su naturaleza transversal, que no permite establecer relaciones causales. Además, el consumo de alcohol de adultos y jóvenes fue autodeclarado. No obstante, hay evidencia que el uso de cuestionarios autodeclarados son un método viable para medir el consumo de alcohol en adolescentes (Engs y Hanson, 1990). Además, la anonimidad y el formato individual de ambos cuestionarios (EDADES y ESTUDES) podría disminuir el sesgo de deseabilidad social inherente a las encuestas. Otra limitación es el uso de proxies para medir las políticas públicas relacionadas con el alcohol, ya que son variables agregadas individuales. A pesar de ello, dichas medidas pueden ser más efectivas para determinar el nivel de cumplimiento de los reglamentos, que la existencia o ausencia de políticas públicas sobre el control del alcohol en cada provincia.

\section{Conclusiones}

Este estudio muestra que el binge drinking entre adolescentes en España está asociado con variables individuales relacionadas con el alcohol, independientemente de las variables contextuales. Además, dichas variables individuales explicaron la variabilidad en el binge drinking entre provincias. Por lo tanto, para ser efectivas, las políticas públicas relacionadas con el control del alcohol deberían ir acompañadas de intervenciones que consideren dichas variables individuales. Prestando especial atención al control parental y a la permisividad respecto al consumo de alcohol por parte de los padres, y a la accesibilidad y disponibilidad de alcohol, por parte de las y los adolescentes. 


\section{Agradecimientos}

Los autores quieren mostrar su agradecimiento a todas las personas que hacen posible la Encuesta sobre uso de drogas en Enseñanzas Secundarias en España (ESTUDES), y especialmente a la Delegación del Gobierno para el Plan Nacional sobre Drogas, que les ha permitido acceder a las bases de datos. Este estudio ha recibido apoyo a través de una ayuda de la Acción Estratégica para la Salud [Ayuda número PI13/00183] y la Red nacional de Trastornos Adictivos [Ayudas números RD12/0028/0018 y RD16/0017/0013]. Este artículo es parte de la tesis doctoral de Ester Teixidó-Compañó, en la Universitat Pompeu Fabra.

\section{Conflicto de intereses}

Los autores declaran la inexistencia de conflicto de intereses, sean de carácter financiero o de otro tipo.

\section{Referencias}

Abar, C., Abar, B. y Turrisi, R. (2009). The impact of parental modeling and permissibility on alcohol use and experienced negative drinking consequences in college. Addictive Behaviors, 34, 542-547. doi:10.1016/j. addbeh.2009.03.019.

Bendtsen, P., Damsgaard, M. T., Huckle, T., Casswell, S., Kuntsche, E., Arnold, P., ... Holstein, B. E. (2014). Adolescent alcohol use: a reflection of national drinking patterns and policy? Addiction, 109, 1857-1868. doi:10.1111/add.12681.

CNAPA (Committee on National Alcohol Policy and Action) (2014). Action Plan on Youth Drinking and on Heavy Episodic Drinking (Binge Drinking) (2014-2016).

Cortés, M. T., Espejo, B., Martín del Río, B. y Gómez, C. (2010). Tipologías de consumidores de alcohol dentro de la práctica del botellón en tres ciudades españolas. Psicothema, 22, 363-368.

Engs, R. y Hanson, D. (1990). Gender differences in drinking patterns and problems among college students: A review of the literature. Journal of Drug and Alcohol Education, 35, 36-47.

Espejo, B., Cortés, M. T., del Río, B. M., Giménez, J. A. y Gómez, C. (2012). Traits that define the different alcohol intensive consume type during the practice of "botellon." Spanish Journal of Psychology, 15, 256-264.

Espelt, A., Mari-Dell'Olmo, M., Penelo, E. y Bosque-Prous, M. (2017). Applied Prevalence Ratio estimation with different Regression models: An example from a crossnational study on substance use research. Adicciones, 29, 105-112. doi:10.20882/adicciones.823.

Foley, K. L., Altman, D., Durant, R. H. y Wolfson, M. (2004). Adults' approval and adolescents' alcohol use. Journal Adolescent Health, 35, 345.e17-26.
Font-Ribera, L., Garcia-Continente, X., Pérez, A., Torres, R., Sala, N., Espelt, A. y Nebot, M. (2013). Driving under the influence of alcohol or drugs among adolescents: The role of urban and rural environments. Accident Analysis Prevention, 60, 1-4. doi:10.1016/j.aap.2013.07.031.

Galán, I., González, M. J. y Valencia-Martín, J. L. (2014). Patrones de consumo de alcohol en España: un país en transición. Revista Española de Salud Pública, 88, 529-540. doi:10.4321/S1135-57272014000400007.

Gómez Fraguela, J. A., Fernández Pérez, N., Romero Triñanes, E. y Luengo Martín, A. (2008). [Alcohol drinking parties and consumption of alcohol and other drugs in youth]. Psicothema, 20, 211-217.

Heimisdottir, J., Vilhjalmsson, R., Kristjansdottir, G. y Meyrowitsch, D. W. (2010). The social context of drunkenness in mid-adolescence. Scandinavian Journal of Public Health, 38, 291-298. doi:10.1177/14034948093 57094.

Hibell, B., Guttormsson, U., Ahlström, S., Balakireva, O., Bjarnason, T., Kokkevi, A. y Kraus, L. (2012). The 2011 ESPAD report. Substance Use Among Students in 36 European Countries (Vol. 36). The Swedish Council for Information on Alcohol and other Drugs (CAN), The European Monitoring Centre for Drugs and Drug Addiction (EMCDDA) and Council of Europe, Cooperation Group to Combat Drug Abuse and Illicit Trafficking in Drugs (Pompidou Group).

Jones, S. C. y Magee, C. A. (2014). The role of family, friends and peers in Australian adolescent's alcohol consumption. Drug and Alcohol Review, 33, 304-313. doi:10.1111/dar.12111.

Jones-Webb, R., Toomey, T., Miner, K., Wagenaar, A. C., Wolfson, M. y Poon, R. (1997). Why and in what context adolescents obtain alcohol from adults: a pilot study. Substance Use and Misuse, 32, 219-228.

Krieg, A. G. y Kuhl, D. C. (2016). Race, Adolescent Binge Drinking, and the Context of Neighborhood Exposure. Deviant Behavior, 37, 615-633. doi:10.1080/01639625.20 15.1060804 .

Krieger, N., Williams, D. R. y Moss, N. E. (1997). Measuring social class in US public health research: concepts, methodologies, and guidelines. Annual Review of Public Health, 18, 341-378.

Kuntsche, E., Rossow, I., Simons-Morton, B., Bogt, T. T., Kokkevi, A. y Godeau, E. (2013). Not early drinking but early drunkenness is a risk factor for problem behaviors among adolescents from 38 European and north american countries. Alcohol Clinical and Experimental Research, 37, 308-314. doi:10.1111/j.15300277.2012.01895.x.

Llorens, N., Barrio, G., Sánchez, A., Suelves, J. M. y ESTUDES Grupo de Trabajo. (2011). Effects of socialization and family factors on adolescent excessive 
drinking in Spain. Prevention Science, 12, 150-161. doi:10.1007/s11121-010-0195-0.

Nash, S. G., McQueen, A. y Bray, J. H. (2005). Pathways to adolescent alcohol use: family environment, peer influence, and parental expectations. Journal of Adolescent Health, 37, 19-28. doi:10.1016/j.jadohealth.2004.06.004.

Nelson, D. E., Naimi, T. S., Brewer, R. D. y Nelson, H. A. (2009). State alcohol-use estimates among youth and adults, 1993-2005. American Journal of Preventive Medicine, 36, 218-224. doi:10.1016/j.amepre.2008.10.018.

Nelson, T. F., Naimi, T. S., Brewer, R. D. y Wechsler, H. (2005). The state sets the rate: the relationship among state-specific college binge drinking, state binge drinking rates, and selected state alcohol control policies. American Journal of Public Health, 95, 441-446. doi:10.2105/AJPH.2004.043810.

Nelson, T. F., Xuan, Z., Babor, T. F., Brewer, R. D., Chaloupka, F. J., Gruenewald, P. J., ... Naimi, T. S. (2013). Efficacy and the strength of evidence of U.S. alcohol control policies. American Journal of Preventive Medicine, 45, 19-28. doi:10.1016/j.amepre.2013.03.008.

Organización Mundial de la Salud (OMS) [World Health Organization]. (2014). Global status report on alcohol and health 2014. Geneva: World Health Organization.

Paschall, M. J., Grube, J. W. y Kypri, K. (2009). Alcohol control policies and alcohol consumption by youth: a multi-national study. Addiction, 104, 1849-1855. doi:10.1111/j.1360-0443.2009.02698.x.

Pedersen, W., Bakken, A. y von Soest, T. (2015). Adolescents from affluent city districts drink more alcohol than others. Addiction, 110, 1595-1604. doi:10.1111/ add. 13005 .

Pettigrew, S., Pescud, M., Jarvis, W. y Webb, D. (2013). Teens' blog accounts of the role of adults in youth alcohol consumption. Journal of Social Marketing, 3, 2840 .

Pitkänen, T., Kokko, K., Lyyra, A.-L. y Pulkkinen, L. (2008). A developmental approach to alcohol drinking behaviour in adulthood: a follow-up study from age 8 to age 42. Addiction, 103 Suppl 1, 48-68. doi:10.1111/ j.1360-0443.2008.02176.x.

Pitkänen, T., Lyyra, A.-L. y Pulkkinen, L. (2005). Age of onset of drinking and the use of alcohol in adulthood: a follow-up study from age 8-42 for females and males. Addiction, 100, 652-661. doi:10.1111/j.13600443.2005.01053.x.

Plan Nacional sobre Drogas. (2016). Encuesta sobre uso de drogas en enseñanzas secundarias en España (ESTUDES), 1994-2014. Ministerio de Sanidad Servicios Sociales e Igualdad.

Rehm, J., Mathers, C., Popova, S., Thavorncharoensap, M., Teerawattananon, Y. y Patra, J. (2009). Global burden of disease and injury and economic cost attributable to alcohol use and alcohol-use disorders. Lancet, 373, 2223-2233.

Reimuller, A., Shadur, J. y Hussong, A. M. (2011). Parental social support as a moderator of self-medication in adolescents. Addictive Behaviors, 36, 203-208. doi:10.1016/j.addbeh.2010.10.006.

Romo-Avilés, N., Marcos-Marcos, J., Marquina-Márquez, A. y Gil-García, E. (2016). Intensive alcohol consumption by adolescents in Southern Spain: The importance of friendship. International Journal of Drug Policy, 31, 138146. doi:10.1016/j.drugpo.2016.01.014

Rossow, I., Keating, P., Felix, L. y McCambridge, J. (2015). Does parental drinking influence children's drinking? A systematic review of prospective cohort studies. Addiction, 111, 204-217. doi:10.1111/add.13097.

Shield, K., Rylett, M., Gmel, G., Rehm, J., Brummer, J. y Møller, L. (2013). Status Report on Alcohol and Health in 35 European Countries 2013. Copenhagen, Denmark: World Health Organization.

van der Vorst, H., Engels, R. C. M. E., Meeus, W., Dekovic', M. y Van Leeuwe, J. (2005). The role of alcohol-specific socialization in adolescents' drinking behaviour. Addiction, 100, 1464-1476. doi:10.1111/j.13600443.2005.01193.x.

Xuan, Z., Blanchette, J. G., Nelson, T. F., Nguyen, T. H., Hadland, S. E., Oussayef, N. L., ... Naimi, T. S. (2015). Youth Drinking in the United States: Relationships with Alcohol Policies and Adult Drinking. Pediatrics, 136, 1827. doi:10.1542/peds.2015-0537.

Xuan, Z., Nelson, T. F., Heeren, T., Blanchette, J., Nelson, D. E., Gruenewald, P. y Naimi, T. S. (2013). Tax policy, adult binge drinking, and youth alcohol consumption in the United States. Alcoholism Clinical and Experimental Research Journal, 37, 1713-1719. doi:10.1111/acer.12152.

Zeigler, D. W., Wang, C. C., Yoast, R. A., Dickinson, B. D., McCaffree, M. A., Robinowitz, C. B., ... Council on Scientific Affairs, American Medical Association. (2005). The neurocognitive effects of alcohol on adolescents and college students. Preventive Medicine, 40, 23-32. doi:10.1016/j.ypmed.2004.04.044. 